Ann. Biol. anim. Bioch. Biophys., I974, 14 (4-A), 581-59o.

\title{
ULTRASTRUCTURE DES SPERMATOZOÏDES DE TAUREAUX DE DIFFÉRENTE FÉCONDANCE. MORPHOLOGIE ULTRASTRUCTURALE, GLYCOPROTÉINES ACROSOMIQUES ET MEMBRANAIRES, CHARGES NÉGATIVES DE SURFACE
}

\author{
J. MORSTIN (1) et M. COUROT \\ avec la collaboration technique de J. L. Courtens \\ Station de Physiologie de la Reproduction, \\ Centre de Recherches de Tours, I. N. R. A., \\ B. P. 1, Nouzilly, 37380 Monnaie
}

\section{RÉSUMÉ}

Du sperme de taureaux de différents niveaux de fécondance a été préparé pour l'observation au microscope électronique. Les techniques mises en œuvre ont permis d'étudier l'ultrastructure générale des spermatozoïdes, les glycoprotéines de l'acrosome, le glycolemme et les charges négatives de surface. Des anomalies ultrastructurales ont été observées dans les différentes parties des gamètes (Pl. I à 3) sur une proportion de spermatozoïdes toujours faible, variable selon les taureaux pour chacun des critères d'analyse retenus. Une estimation semi-quantitative de ces anomalies montre qu'il y a une plus grande proportion d'anomalies des spermatozoides chez les taureaux de moins bonne fécondance (tabl. I et 2). Les plus importantes et sans doute les plus reliées à la fécondance portent sur le noyau, l'acrosome et les mitochondries. Certaines comme l'irrégularité des charges négatives de surface semblent ne pas avoir de liaison nette avec la fécondance.

\section{INTRODUCTION}

La variabilité de fécondance entre taureaux est connue. Elle ne peut être éliminée en dépit des nombreux tests de contrôle appliqués lors de la récolte et de la préparation de la semence pour ne conserver que les meilleurs éjaculats (SALrSBury

(1) Institut de Génétique et d'Élevage de l'Académie Polonaise des Sciences, Jastrzebiec, 05-55 I Mrokow. Boursier du Gouvernement Français pendant son séjour au laboratoire de l'I. N. R. A. 
et VAN Demark, I96r). Des imperfections non décelables par les tests morphologiques ou métaboliques courants sont vraisemblablement à l'origine de la subfertilité de certains taureaux. Pour étudier ce problème, nous avons entrepris l'observation en microscopie électronique des spermatozoïdes de taureaux considérés comme normaux ou comme subféconds, d'après leurs résultats de non-retours en insémination artificielle. Compte tenu de l'importance de la membrane plasmique et de l'acrosome au moment du contact des gamètes lors de la fécondation, nous avons spécialement porté notre attention sur ces structures sans toutefois négliger les autres éléments du spermatozoïde.

\section{MATÉRIEL ET MÉTHODES}

\section{1. - Origine du sperme}

Io à 25 doses provenant de un ou deux éjaculats de 25 taureaux appartenant à trois centres d'insémination artificielle français ont servi pour ce travail. La répartition des animaux selon les races et leur fécondance, jugée par rapport à la moyenne du centre auquel ils appartiennent, est donnée dans le tableau I (1). La semence était diluée, conditionnée et congelée pour usage en insémination artificielle selon les techniques retenues par chaque centre. Les dilueurs et conditionnements suivants ont été utilisés : lait - jaune d'œuf, paillettes moyennes ; jaune d'œuf - acide caproïque - tampon glycocolle - citrate - glucose, paillettes fines; citrate - jaune d'œuf, paillettes fines ou moyennes; jaune d'œuf - lactose, pastilles - sans que cela semble avoir une influence particulière sur les observations en microscopie électronique.

\section{TABLEAU I}

Répartition des taureaux selon la race et la fécondance (1) Qualité des éjaculats utilisés dans ce travail $\left({ }^{2}\right)$

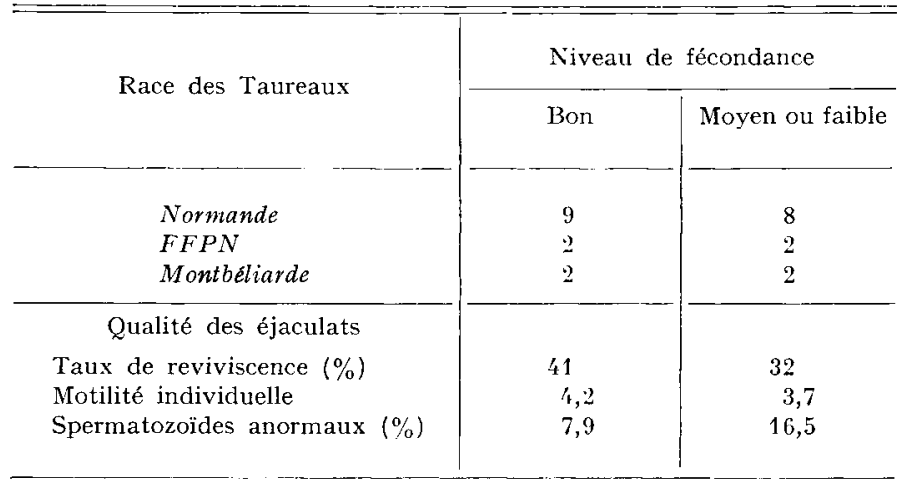

(1) Fécondance jugée par rapport à la moyenne du centre auquel appartiennent les taureaux. La fécondance de chacun des taureaux et le nombre d'I. A. premières sur lequel elle a été calculée sont données dans les tableaux 2 et 3 .

(2) La qualité des éjaculats a été appréciée au dégel par observation des spermatozoïdes mobiles (reviviscence et motilité) et sur frottis colorés à l'éosine (anormaux).

(1) Ce tableau précise en outre la qualité des éjaculats utilisés dans ce travail par le taux de reviviscence, la motilité individuelle et le pourcentage de spermatozoïdes porteurs d'anomalies morphologiques visibles en microscopie photonique. 


\section{2. - Préparation des échantillons pour observation}

a) Fixation.

Un volume de semence, paillettes ou pastilles, est décongelé à température ambiante dans cinq volumes du mélange fixateur mis au point par CourTens (1973) : glutaraldéhyde 2,7 p. Ioo dans du tampon cacodylate $(0,053 \mathrm{M})$ additionné de glycérol $(\mathrm{I}, 46 \mathrm{M}), \mathrm{KCl}(0, \mathrm{I} 8 \mathrm{M}), \mathrm{CaCl}_{2}$ $(0,0$ I $2 \mathrm{M})$ et $\mathrm{MgCl}_{2}\left(0,0{ }_{4} \mathrm{M}\right)$. Après 45 à 60 minutes de fixation le surnageant est éliminé par centrifugation et les spermatozoïdes sont post-fixés par une solution de glutaraldéhyde à $\mathrm{I}$ p. Ioo dans du tampon cacodylate $0,2 \mathrm{M}$, pendant 60 minutes à température ambiante. Aucun traitement à l'acide osmique n'est appliqué en raison des colorations ultérieures. Les spermatozoïdes sont ensuite rincés dans le tampon additionné de sucrose $(0, \mathbf{I} M)$ et subdivisés en trois fractions selon les traitements de coloration qui doivent être appliqués.

\section{b) Coloration.}

- Pour l'observation de structure générale, les spermatozoïdes déshydratés à l'éthanol sont inclus dans le milieu de Spurr (1969) et les coupes contrastées par l'acétate d'uranyle et le citrate de plomb (Venable et Coggeshall, I965).

- Pour la mise en évidence des groupes acides sur la membrane plasmique (charges négatives), les spermatozoïdes sont traités à l'acide acétique I $2 \mathrm{p}$. xoo pendant 20 minutes puis par l'hydroxyde de fer colloïdal chargé positivement d'après Mowry (I958) avant inclusion dans le milieu de Spurr.

- Pour l'étude des glycoprotéines, les spermatozoïdes inclus dans le glycol-méthacrylate (GMA) sont colorés sur coupes par l'acide phosphotungstique dans l'acide chlorhydrique normal (APT/HCl N) d'après PEASE (1970), technique spécifique des glycoprotéines (RAmbourg, I973).

La semence de 6 à 12 taureaux a été préparée simultanément. Les observations des préparations - codées pour éviter que l'observateur soit influencé - sont réalisées sur un microscope Philips EM 300 à des grossissements directs de Io ono à 50 ooo fois.

\section{RÉSULTATS}

\section{I. - Spermatozoïdes normaux}

Les techniques employées sont satisfaisantes; elles ont permis d'observer les images classiques de l'ultrastructure des spermatozoïdes normaux au niveau de la tête, de la pièce intermédiaire et du flagelle. Ces images ont servi de référence pour définir les anomalies rencontrées sur certains spermatozoïdes. En outre, il est apparu après fixation au glutaraldéhyde seul et coloration classique, qu'une couche continue de matériel périnucléaire protéique existe tout autour de la tête du spermatozoïde entre l'acrosome et le noyau ainsi qu'entre la zone dense post-acrosomique et le noyau. A son extrémité antérieure, ce matériel se condense pour former le perforatorium (fig. 2). Par ailleurs, le glycolemme est normalement réparti en une couche continue et régulière sur toute la surface du spermatozoïde (fig. I2 à I5). Il en est de même des charges négatives de surface (fig. 2I à 24).

\section{2. - Spermatozoides anormaux}

Chez presque tous les taureaux, des anomalies ultrastructurales invisibles en microscopie photonique ont été observées sur une certaine proportion des spermatozoïdes, variable selon les animaux. Plusieurs types d'anomalies peuvent être ren- 
contrés chez le même taureau : dans les préparations pour l'étude de la " structure générale ", elles concernent la morphologie, anormale par la forme ou l'aspect, d'éléments déterminés des spermatozoïdes. Pour les glycoprotéines de l'acrosome ou du glycolemme et pour les charges négatives de surface de la membrane du spermatozoïde, l'homogénéité et l'importance du matériel réactif ont été appréciées, les images les moins fréquentes étant considérées comme anormales.

\section{a) Observations.}

Au niveau de la tête des spermatozoïdes, des aberrations ont été rencontrées sur le noyau, l'acrosome et la membrane plasmique. Chez certains taureaux, le noyau présente des invaginations de l'enveloppe nucléaire et du matériel périnucléaire qui la recouvre formant ainsi une cavité plus ou moins fermée (fig. I $a$ et $b$ ) qui peut atteindre une grande taille: jusqu'à $5 \mu$ dans sa plus grande dimension. Dans d'autres cas, l'acrosome apparaît anormal de forme ou de structure, avec du matériel granu-

TABLEAU 2

Anomalies ultrastructurales des spermatozoides chez les taureaux de bonne fécondance

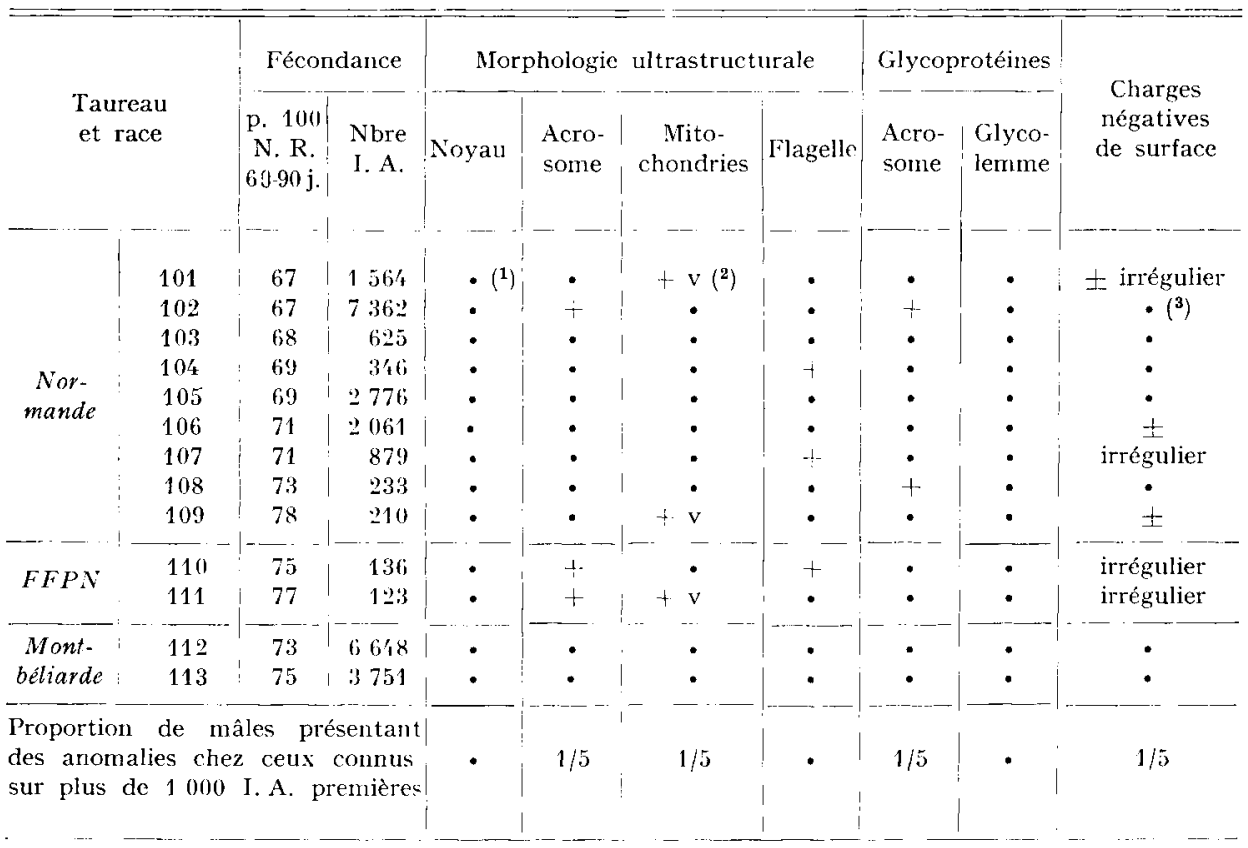

(1) (•): Pratiquement pas d'anomalies pour la partie considćrée ;

(+) : Anomalies présentes, mais en très faible proportion : < 1 p. 100 des spermatozoïdes; $(++)$ : Anomalies plus fréquentes : 1 à 5 p. 100 des spermatozoïdes.

(2) Pour les mitochondries, on rencontre quelcues anomalies de structure : mitochondries "vides" (v) (fig. 4 ).

$\left({ }^{3}\right)$ Les charges de surface peuvent être normales $(\cdot)$, faibles $\left(t_{-}\right)$et/ou irrégulièrement réparties (irrégulier). 
leux à son extrémité antérieure (fig. 2) ou bien des irrégularités de distribution (fig. I6 et I7), voire même une très faible densité, du constituant glycoprotéique sans que la taille de l'acrosome paraisse modifiée. La membrane plasmique présente quelquefois autour de la tête du spermatozoïde un glycolemme fragmenté (fig. I6 à I8) au lieu d'être normalement continu (fig. I I à I3). Dans quelques cas enfin, les charges négatives de surface sont plus faibles (fig. 25) que la normale (fig. 2 I à 23), ou même presque inexistantes.

Le flagelle peut aussi être porteur d'anomalies de structure dont les plus fréquentes concernent les mitochondries. Celles-ci sont quelquefois de taille irrégulière (fig. 3), en surnombre ou en position anormale dans l'hélice (fig. 3) ; la structure de certaines d'entre-elles apparaît vide (fig. 4) ou " anguleuse " (fig. 5). Ceci n'est pas un artefact de fixation puisque, dans les mêmes préparations, on observe des spermatozoïdes porteurs de ces anomalies et des spermatozoïdes tout à fait comparables à ceux des descriptions classiques de la littérature. Le flagelle présente aussi quelques anomalies beaucoup plus rares sous la forme de cavités assez allongées (fig. 8) limitées par une membrane, qui se rencontrent à tous les niveaux depuis le cou (fig. 7) jusqu'à l'extrémité du flagelle (fig. 9). On observe très rarement des irrégularités de l'axonème telles que des doublets en surnombre (fig. ro). Le glycolemme, normalement continu et régulier tout au long du flagelle (fig. I4 et I5) apparait quelquefois très ténu et comme en pointillé (fig. I9 et 20 ). Les charges négatives de surface peuvent aussi être très faibles alors qu'en conditions normales elles sont très denses (fig. 23 et 24), autant que sur la membrane de la tête du spermatozoïde.

\section{b) Relation avec la fécondance.}

Pour chaque échantillon, la proportion des anomalies rencontrées a été estimée semi-quantitativement $:<x$ p. Ioo et $\mathrm{I}$ à $5 \mathrm{p}$. Ioo. De plus, pour chacune, on a apprécié l'intensité de son développement suivant les cas rencontrés. C'est pourquoi il est pratiquement impossible de donner une expression chiffrée précise des résultats.

Lorsque les résultats sont rapportés par taureau, et les taureaux groupés selon leur niveau de fécondance (tabl. 2 et 3 ), il apparaît qu'il y a une plus grande proportion d'anomalies des spermatozoides chez les taureaux de moins bonne fécondance. Aucun de ces animaux n'est exempt d'une atteinte notable (en nombre de spermatozoïdes touchés) dans l'un ou l'autre, voire même souvent dans plusieurs des constituants du gamète, noyau, acrosome, mitochondries, membrane... alors que quatre taureaux du groupe de bonne fécondance n'ont pratiquement rien d'anormal. Par ailleurs, on ne voit d'atteintes graves du noyau, d'anomalies nombreuses de l'acrosome ou des mitochondries, d'absence des charges de surface de la membrane plasmique que chez les taureaux de fécondance faible ou moyenne (tabl. 3).

Toutefois, il n'apparaît pas une répartition univoque des anomalies puisque même de bons géniteurs présentent des figures anormales de leurs spermatozoides (tab1. 2). Mais elles restent rares et, prises séparément, ne sont donc pas incompatibles avec une qualité fécondante normale du sperme. Certaines, plus fréquentes, telles les cavités dans le flagelle et 1a répartition irrégulière des charges négatives de surface observées dans les deux groupes d'animaux, semblent indépendantes de la qualité du sperme. 
TABLEAU 3

Anomalies ultrastructurales des spermatozoides chez les taureaux de fécondance moyenne ou faible.

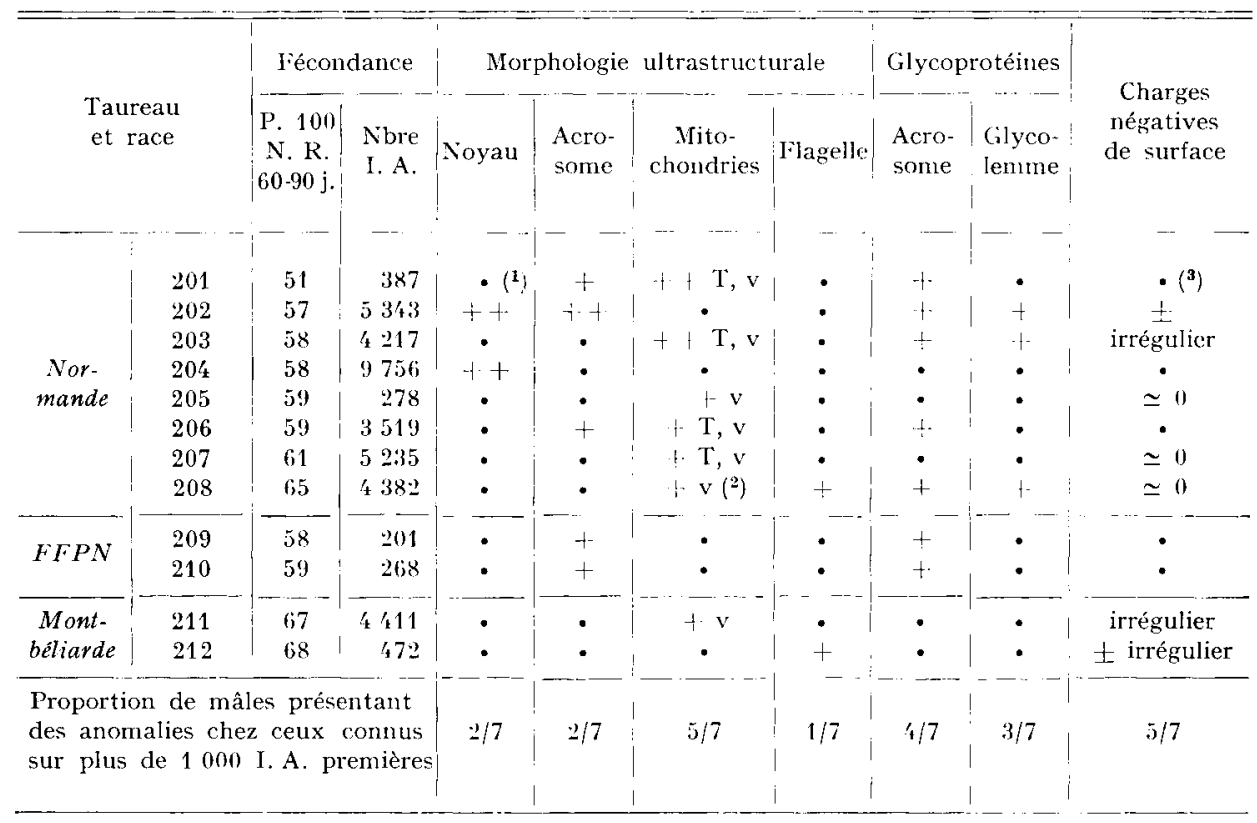

(1) (•) : Pratiquement pas d'anomalies pour la partie considérée ;

(十) : Anomalies présentes, mais en très faible proportion : $<1$ p. 100 des spermatozoïdes ;

$(++)$ : Anomalies plus fréquentes : 1 à 5 p. 100 des spermatozoïdes.

(2) Pour les mitochondries, on rencontre des anomalies de taille ( $T$ ) ou de structure : mitochondries "vides" (v) (fig. 3 et 4 ).

$\left({ }^{3}\right)$ Les charges de surface peuvent être normales $(\bullet)$, irrégulièrement réparties (irrégulier), faibles $( \pm$ ) ou presque inexistantes $(\simeq 0)$.

\section{DISCUSSION}

\section{I. - Structure normale des spermatozoides}

Ce travail sur 1'ultrastructure des spermatozoïdes de taureau précise quelques informations sur la cellule normale qui avaient été peu soulignées jusqu'à maintenant. Il rapporte la présence du matériel périnucléaire prolongeant le perforatorium tout autour du noyau du spermatozoïde, sous l'acrosome et la lame post-acrosomique. Si la présence du perforatorium n'est pas discutée chez le taureau, le matériel périnucléaire n'y était reconnu que sous le segment équatorial (BLom et BIRCH-ANDERSEN, I96I ; NICANDER et BANE, I966 ; KANN, I969; WoODING et O'DONNELI, I97I) et non à la surface de la plus grande partie du noyau, fossette d'implantation exceptée comme dans d'autres espèces (JONES, I970; FLECHON, I973; LALII, I973). 
Par ailleurs, la présence d'un glycolemme, couche glycoprotéique continue sur toute la surface du spermatozoïde normal, est démontrée. Ceci complète les premières observations de KANN et HoLLANDE (I972) pour qui cette couche semblait discontinue, mais différencie les spermatozoïdes du taureau et du lapin où la pièce intermédiaire est dépourvue de glycolemme (FLECHON, I973). La même technique de coloration met en évidence les glycoprotéines de 1'acrosome, même dans le segment équatorial, confirmant les résultats de KANN et Hor, ANDE (I972); elle distingue ainsi les spermatozoïdes du taureau et ceux du lapin chez qui cette partie de l'acrosome réagit très peu avec 1'AP'T/HCl N (FLECHON, I973). A propos de l'acrosome, nous devons dire, en accord avec Woodrng et O'DonNeLL (I97I), qu'il n'y a normalement pas de vacuole dans l'extrémité antérieure de l'acrosome contrairement aux affirmations de Blom et BrRCr-ANDERSEN (I96I) et de KANN (I969). A l'endroit de l'épaississement marginal, on note une structure hétérogène qui ne prend pas le colorant des glycoprotéines (fig. I2) ; elle correspond aux corps denses décrits chez le lapin (FLECHON, I973).

Enfin, nous avons retrouvé la répartition assez homogène des charges négatives de surface sur le spermatozoïde éjaculé du taureau, déjà signalée par YANAGMACHI et al. (I972) à la différence de nombreux rongeurs où ces charges sont faibles sur le flagelle (ibid.).

\section{2. - Anomalies ultrastructurales}

Nous n'avons pu donner qu'une évaluation semi-quantitative des anomalies ultrastructurales, car les techniques employées n'avaient pas permis d'orienter les spermatozoïdes en cours d'inclusion comme l'a depuis proposé JonEs (I973). Aussi, nous n'avons pu en faire une étude quantitative précise; cependant, la fréquence des anomalies observées reste toujours faible. A ce propos, il convient de rappeler que ce travail a porté sur le sperme de taureaux en service dans des centres d'insémination artificielle, donc de géniteurs déjà triés sur leurs capacités à donner du sperme convenable. De plus, les éjaculats de ces taureaux sont tous contrôlés par les techniciens des centres d'I. A. et seuls sont conservés ceux qui ont un taux élevé de reviviscence et une motilité individuelle des spermatozoïdes convenable. Une vérification faite au laboratoire sur les doses utilisées dans ce travail a montré qu'il en était bien ainsi (tabl. I). C'est donc sur une population d'éjaculats très sélectionnés qu'ont porté nos observations. Cependant, le pourcentage des spermatozoïdes anormaux, déterminé sur frottis par microscopie classique était plus élevé chez les taureaux de moyenne fécondance (I6,5 p. I00) que chez les bons taureaux (7,9 p. Ioo, tabl. I) ; et, à l'échelle ultrastructurale, un certain nombre d'anomalies a été observé qui, même si il est faible, peut rendre compte de la moins bonne aptitude à la fécondance de certains taureaux. Un exemple est déjà connu d'un taureau pratiquement stérile avec seulement I,73 p. I ooo spermatozoïdes diploïdes dans l'éjaculat (ESNAULT et OrTAVANT, I967).

On peut donc envisager que certaines des anomalies observées dans ce travail peuvent à elles seules rendre compte de la mauvaise qualité fécondante de certains taureaux. Ce doit être le cas des invaginations de l'enveloppe nucléaire observées chez 202 et 204 (tabl. 3) comparables à celles décrites par Hrudra et ZibriN (ig64), SAacke et Almouis'i (I964), et BANE et Nicander (I965) dont ces derniers 
auteurs disent qu'elles accompagnent une sévère atteinte de la spermiogenèse. De telles anomalies ne sont pratiquement pas remarquées chez les bons taureaux (tab1. 2). Deux autres constituants des spermatozoïdes, l'acrosome et les mitochondries, présentent aussi une proportion importante d'anomalies chez les moins bons taureaux (tabl. 3). Enfin, c'est seulement dans ce même groupe d'animaux que l'absence quasicomplète de charges négatives de surface sur un nombre important de spermatozoïdes est observée (tab1. 3). Compte tenu de ce que la semence de plusieurs animaux pris au hasard a été traitée simultanément, tant au cours de la préparation dans les centres d'I. A. que pour les techniques de microscopie électronique au laboratoire, on ne peut invoquer des artefacts pour expliquer les résultats observés ; en effet, il n'apparaît pas de liaison avec le dilueur, la méthode de refroidissement ou les techniques de fixation et de coloration. Les anomalies observées sont donc bien caractéristiques de la semence des taureaux et il apparaît que certaines sont notablement plus fréquentes chez les taureaux de basse fécondité. Leur recherche plus systématique permettrait peut-être d'éliminer de la reproduction un certain nombre de géniteurs dont la carrière serait médiocre.

Reçu pour publication en mars 1974.

\title{
REMERCIEMFNTS
}

Ce travail a été réalisé pendant le séjour du premier auteur (J. M.) à la Station de Physiologie de la Reproduction grâce à une bourse du Grouvernement français auquel M. MorstıN adresse ses remerciements. En outre, ce travail a été possible du fait de la collaboration des centres d'insémination artificielle qui ont accepté de mettre à notre disposition la semence de taureaux choisis selon leur niveau de fécondance. Nous remercions très vivement MM. JondET, ChEvaldonne et VuIDEPOT, responsables de ces organismes. Nous remercions également M. FLÉchon, lab. microscopie électronique, I. N. R. A. Jouy en Josas, qui nous a initiés à la pratique de certaines techniques d'histochimie ultrastructurale.

\section{SUMMARY}

\author{
FINE STRUCTURE OF SPERMATOZOA OF BULLS OF DIFFERENT FERTILITY \\ (ULTRASTRUCTURAI, MORPHOLOGY, GLYCOPROTEINS OF THE ACROSOME \\ AND OF THE CELI, MEMBRANE, NEGATIVE SURFACE CHARGES)
}

Sperm samples from bulls of different fertilizing ability in use in A. I. centers were processed for E. M. study of the cell morphology (glutaraldehyde fixation, uranyl-lead staining), the glycoproteins (glycol methacrylate embedding and APT/HCl N staining) and the negative charges of the cell membrane (colloidal iron hydroxyde staining).

Ultrastructural abnormalities were observed (Plates I to 3) on a low percentage of spermatozoa in most of the bulls. A semi-quantitative analysis shows a higher proportion of gamete abnormalities in the sperm of bulls of low fertility (tables 2 and 3). Possibly the most relevant to low fertility level are those concerning the nucleus, the acrosome and the mitochondria. The irregular distribution of the negative charge on the spermatozoa seems unrelated to fertilizing abitlity of the male gamete. 


\section{RÉFÉRENCES BIBLIOGRAPHIQUES}

Bane A., Nicander L., I965. Pouch formations by invaginations of the nuclear envelope of bovine and porcine sperm as a sign of disturbed spermiogenesis. Nord. Vet. Med., 17, 628-632.

Blom E., Birch-ANDERSEN A., 1965. The ultrastructure of the bull sperm. II. The sperm head. Nord. Vet. Med., 17, 193-212.

Courtens J. L., 1973. (Communication personnelle).

Esnault C., Ortavant R., i967. Origine des spermatozoïdes diploïdes présents dans l'éjaculat d'un taureau Charolais. Ann. Biol. anim. Bioch. Biophys., 7, 25-28.

FLÉCHON J. E., I973. Ultrastructural and cytochemical modifications of rabbit spermatozoa during epididymal transport. In Sperm transport, survival and fertilizing ability E. S. E. HAFEZ and C. THIBAUlt ed. Colloque I. N.S.E.R.M., 26, II5-I40.

HRUDKA F., ZIBRIN M., I964. The incidence of vacuoles in the nucleoplasma of normal bull spermia. Vet. Med. (Prague), 37, I I-I8.

Jones R. C., I97o. Studies of the ultrastructure of the head of some mammalian spermatozoa with particular reference to the perforatorium. VII Cong. intern. Micr. Electr., Grenoble, 64I-642.

Jones R. C., 1973. Preparation of spermatozoa for electron and light microscopy. J. Reprod. Fert., 33, I45-I49.

Kans M. L., I969. Étude de l'ultrastructure du complexe acrosomique du spermatozoïde de taureau avant et après congélation. C. R. Acad. Sc., Paris, Ser. D., 269, 2026-2028.

Kann M. L., Hollande E, 1972. Étude cytochimique du glycolemme et de la rćgion postacrosomique du spermatozoỉde de taureau, avant et après congélation. C. R. Acad. Sc., Paris, Ser. D, 274, 295-298.

LALLI M. F., I973. The head of the rat spermatozoa and its formation during spermiogenesis : an electron microscopic study. Diss, Abst., 33, 2900.

Mowry R. W., r958. Improved procedure for the staining of acidic polysaccharides by Müller's colloidal (hydrons) ferric oxide and its combination with the feulgen and the PAS reactions. Lab. invest., 7, 566-576.

NicANDER L., BANE A., Ig66. Fine structure of the sperm head in some mammals, with particular reference to the acrosome and the subacrosomal substance. Z. Zellforsch., 72, 496-515.

PeAsE D. C., r97o. Phosphotungstic acid as a specific electron stain for complex carbohydrates. J. histochem. Cytochem., 18, 455-458.

Rambourg A., I973. Techniques de détection des glycoprotéines en microscopie électronique. Coll. Intern. Histochimie, Tours (sous presses).

SaAcke R. G., Almouist J. O., 1964. Ultrastructure of bovine spermatozoa. The head of normal, ejaculated sperm. Amer. J. Anat., 115, I $43^{-I} 62$.

Salisbury G. W., Vandemark N. L., I96r. Physiology of reproduction and artificial insemination. of cattle. Freeman and Co. Edt.

SPURR A. S., I969. A low-viscosity epoxy resin embedding medium for electron microscopy. $J$. Ultrastruc. Res., 26, $3 \mathrm{I}-43$.

Venable J. H., Coggeshall R., 1965. A simplified lead citrate stain for use in electron microscopy. J. Cell. Biol., 25, 407-408.

Wooding F. B. P., O'DonNell J. M., ig7I. A detailed ultrastructural study of the head membranes of ejaculated bovine sperm. J. Ultrastruc. Res., 35, $7 \mathrm{I}-85$.

Yanagimachi R., Noda Y. D., Fujimoto M., Nicolson G. L., 1972. The distribution of negative surface charges on mammalian spermatozoa. Amer. J. Anat., 135, 497-520. 
PLANCHE I

Diverses anomalies de structure observées dans les spermatozoïdes de taureaux

Inclusion : Spurr; Coloration : Uranyl-Plomb ( $\times$ 40 000).

$\mathrm{r} a$ et $b:$ Anomalies nucléaires. Invagination de l'enveloppe nucléaire et du matériel cytoplasmique qui la recouvre dans la région de l'acrosome (I $a$, coupe longitudinale) et de la lame postacrosomique ( $\mathrm{I} b$, coupe transversale).

2 : Anomalie de l'acrosome. Présence de matériel granuleux au lieu d'un matériel normalement homogène à côté du corps dense.

3 à 7 : Anomalies des mitochondries. Répartition et taille irrégulière (3); hétérogénéité de structure (4) et de forme (5). Aspect de référence avec la technique utilisée (6).

8 à II : Anomalies du flagelle et de l'axonème. Cavités entourées d'une membrane à différents niveaux du flagelle : dans la zone proximale de la pièce intermédiaire $(7)$; dans la pièce principale (8, coupe longitudinale) ou à son extrémité (9). Doublets périphériques en surnombre : I I au lieu de 9 dans la pièce intermédiaire (IO). 

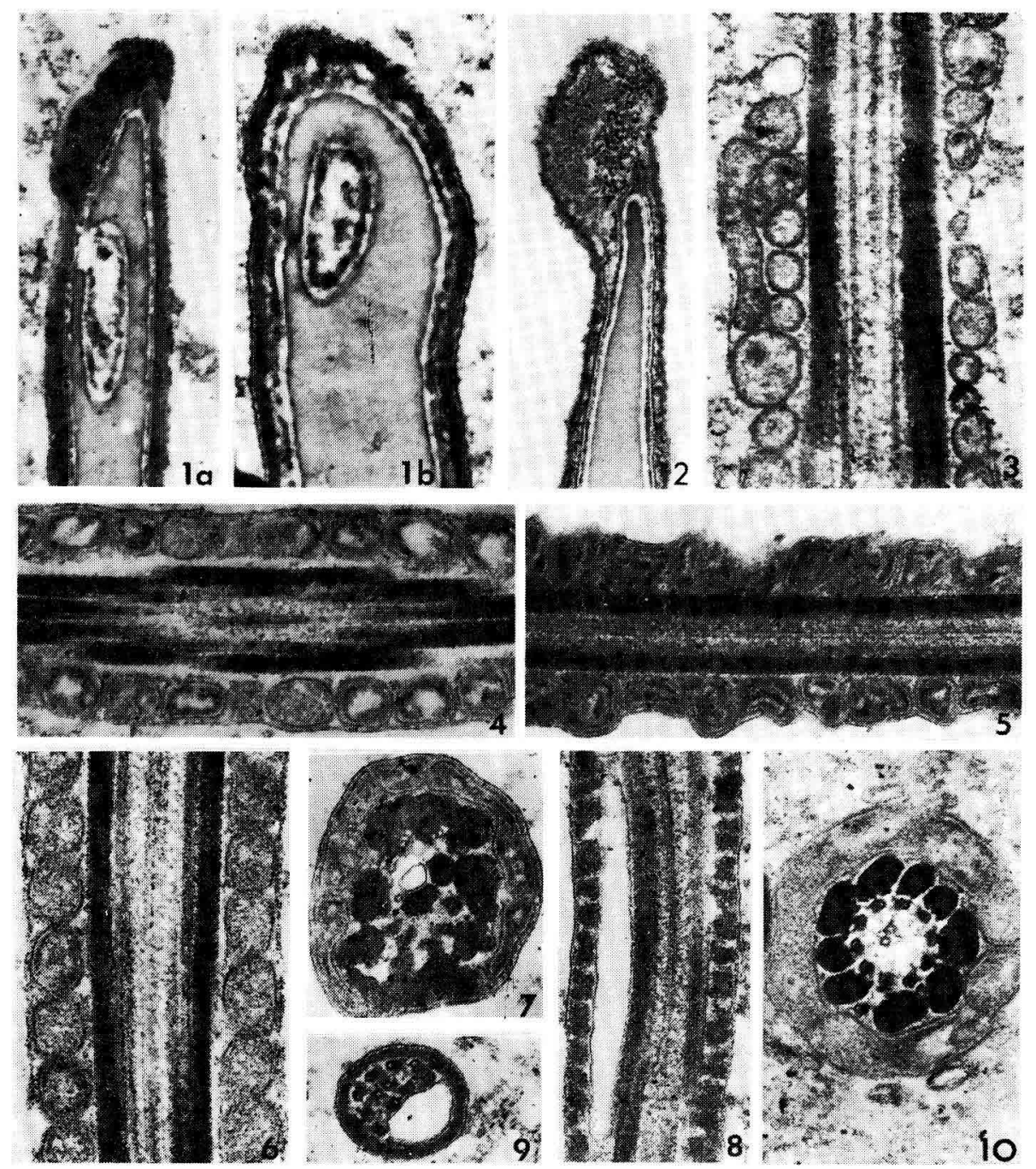

J. MORSTIN et M. COUROT 


\section{PLANCHE 2}

Glycoprotéines de l'acrosone et GLY ColenMe DES SPERMATOZOÏDES DE TAUREAU

Inclusion : GMA. Coloration APT/HCl N. $\times 40.000$.

11 à 15 : Aspect normal au niveau de la tête en coupe transversale (11), ou longitudinale dans la zone antérieure (12) et postérieure (13), coupe transversale de la pièce intermédiaire (14) ct coupe longitudinale de la pièce principale (15).

16 à 20 : Irrégularité de la répartition des glycoprotéines de l'acrcsome (16-17) et du glycolemme à différents endroits du spermatozoïde : tête (18), pièce intermédiaire (19) et pièce principale (20). En outre, la figure 16 allie une morphologie anormale de l'acrosome et une répartition irrégulière des glycoprotéines. 


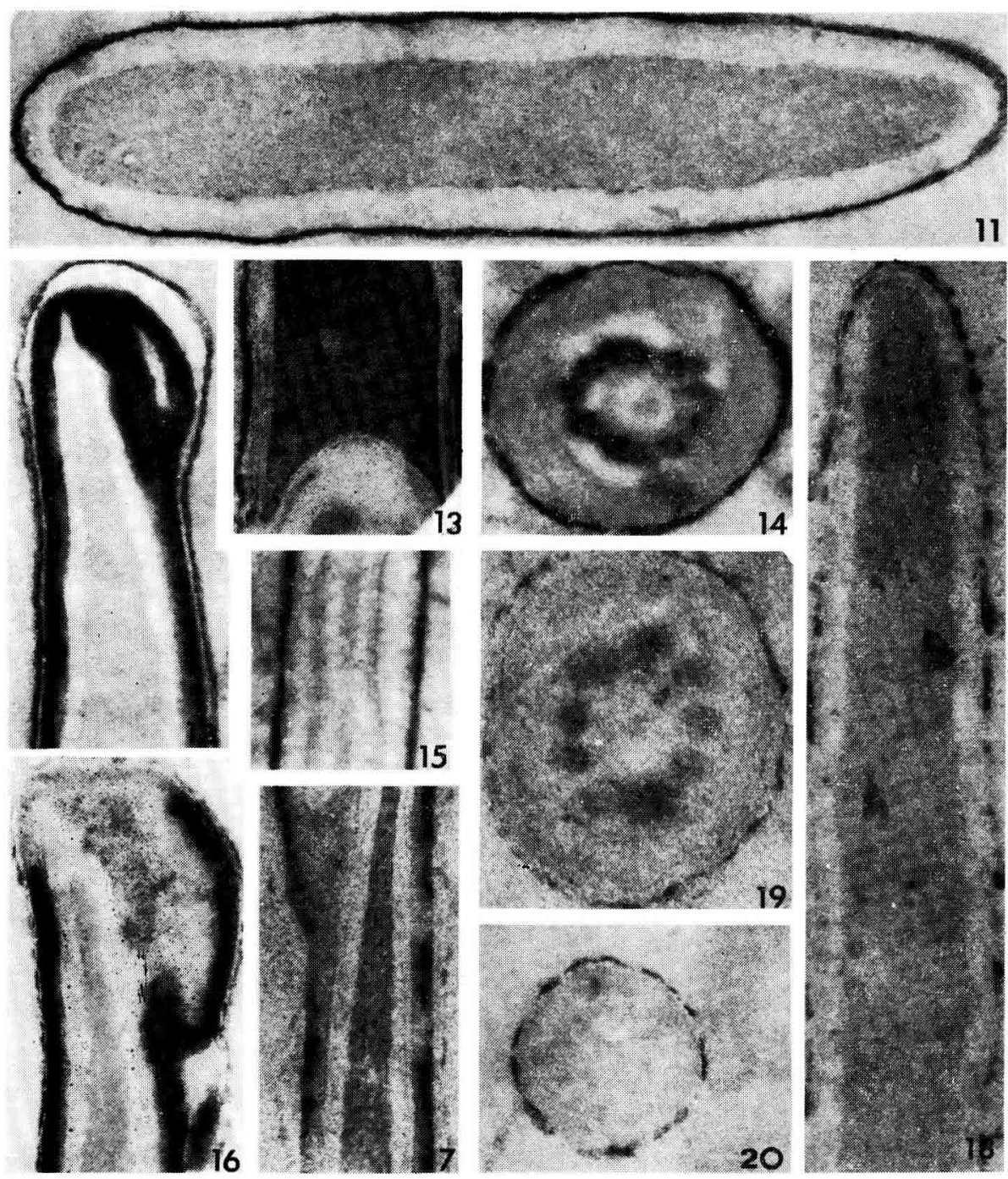

J. MORSTIN et II. COURO'T 
I'LANC:HE 3

Charges négatives de SURFace

DES SPERMATOZOÏDES DE TAUREAU

Inclusion : SPURR. Coloration : hydroxyde de Fer colloïdal chargé positivement. $\times 40.000$.

21 à 24 : Aspect normal avec répartition à peu près aussi importante sur la tête (21-22-23), la pièce intermédiaire (23) et la pièce principale (24).

25: Charges beaucoup plus dispersées sur la tête d'un spermatozoïde. A l'extrême, certains spermatozoïdes apparaissent presque complètement démunis de charges négatives de surface. 


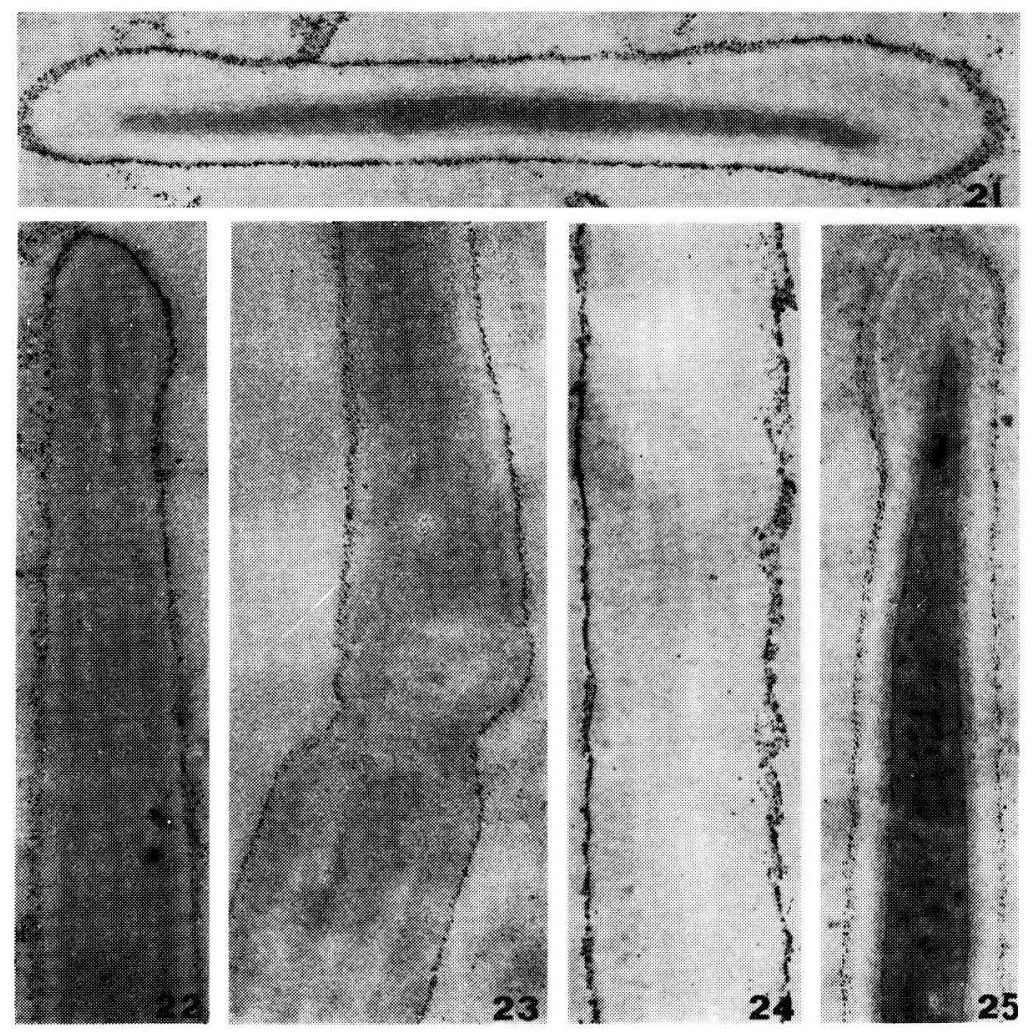

